\title{
Contenido de mercurio en músculo de algunas especies ícticas de consumo presentes en el río Quito, sistema hídrico del río Atrato, Chocó, Colombia
}

\section{Mercury content in muscle of some fish species present in the Quito River, water system of the Atrato River, Chocó, Colombia}

\section{Yiskar Damián Murillo Asprilla*}

\section{Resumen}

En Colombia la minera auroplatinifera es la principal causa de contaminación de los ambientes acuáticos. El mercurio (Hg) es el elemento central para obtener el metal con valor comercial; es el responsable de los efectos acumulativos en la fauna íctica que a menudo es utilizada por las comunidades de las riberas. Mediante técnicas artesanales con trasmallos y atarraya se capturaron 10 especies ícticas en el río Quito. Se utilizó un proceso de espectrometría de absorción atómica de vapor en frío y se determinaron los niveles de mercurio presente en el músculo de cada muestra. Los especímenes analizados mostraron que contienen Hg total en sus músculos; el grupo de los bagres arrojó los más altos valores, siendo Pseudopimelodus schultzi $(0,76 \mu \mathrm{g} / \mathrm{g})$ y Pimelodella chagresii $(0,62 \mu \mathrm{g} / \mathrm{g})$, las especies con mayores niveles de mercurio; por su parte Trachelypterus fisheri $(0,17 \mu \mathrm{g} / \mathrm{g})$, y Sturisomathictys tamanac $(0,17 \mu \mathrm{g} / \mathrm{g})$, tuvieron los valores más bajos. En general los análisis muestran una vez más que las especies de los eslabones más altos en la cadena trófica son los organismos que biogmanifican mejor el $\mathrm{Hg}$.

Palabras clave: Actividad minera, Íctiofauna, Mercurio en río Quito.

\begin{abstract}
Currently, the Atrato River basin is subjected to strong anthropic pressures, especially gold and platinum mining, in which mercury is used as the main element to obtain the metal with commercial value (gold). Is responsible for cumulative effects to the fish fauna that is often used by riverbank communities. Through artisanal techniques with trammel net and cast net, 10 fish species were captured in the Quito River. A cold vapor atomic absorption spectrometry process was used to determine
\end{abstract}

* Magister (C) Ciencias Biológicas, Facultad de Ciencias Básicas, Universidad de la Amazonia, Florencia, Caquetá.

e-mail: ydmurillo@iiap.org.co 
the levels of mercurypresent in the muscle of each sample. The specimens analyzed showed total hg in their muscles, the group of catfish showed the highest values of hg, being Pseudopimelodus schultzi $(0.76 \mu \mathrm{g} / \mathrm{g})$ and Pimelodella chagresii $(0.62 \mu \mathrm{g} / \mathrm{g})$, the species with higher levels of mercury; on the other hand, Trachelypterus fisheri $(0.17 \mu \mathrm{g} / \mathrm{g})$, and Sturisomathictys tamanac $(0.17$ $\mu \mathrm{g} / \mathrm{g})$, reported the lowest values. In general, the analyzes show once again that the species of the highest links in the trophic chain are the organisms that best biogmanise hg.

Keywords: Icthyofauna of Quito River, Mercury, Mining activity.

\section{Introducción}

El mercurio (Hg) es un metal altamente tóxico con un alto potencial de bioacumulación en peces y en general, en organismos acuáticos. En consecuencia, el consumo de productos hidrobiológicos se considera la principal ruta de exposición de los seres humanos a este contaminante (WHO 2007). El Hg ingresa a la atmósfera y a los ecosistemas acuáticos como resultado de fenómenos naturales como vulcanismo y erosión; sin embargo, la principal causa del incremento de los ambientes naturales con $\mathrm{Hg}$ son las actividades antrópicas (Gustin et al. 2000). La presencia de $\mathrm{Hg}$ en los tejidos de la biota está directamente relacionada con la minería aurífera (Lechler et al. 2000), la industria química y el empleo de combustibles fósiles (Limbong et al. 2003).

En Colombia, una de las principales causas de deterioro de las fuentes hídricas en los llamados distritos mineros está relacionada con la minería aluvial a cielo abierto, debido a que el agua es un insumo indispensable en el proceso productivo minero. Su uso es más intensivo en la fase de producción, donde se combina con reactivos químicos como $\mathrm{Hg}$, para separar el metal de la roca y así obtenerlo con valor comercial (Herrera y Millones 2011).

Una de las formas y quizás la más utilizada para evaluar el estado de contaminación por $\mathrm{Hg}$ en los cuerpos de agua de todo el mundo, se centraliza en el uso de las poblaciones ícticas, debido a que son los mejores organismos adaptados a los hábitats acuáticos, lo que les ha permitido presentar todos los niveles tróficos, además, sirven como base alimenticia para muchas comunidades humanas (Bourgoin y Quiroga 2002, Hylander et al. 2000, Barbosa et al. 2003, Da Silva et al. 2005). En un medio acuático contaminado por $\mathrm{Hg}$ los peces experimentan un proceso de bioacumulación que corresponde al incremento de su concentración en los tejidos a lo largo de la vida, por lo que se genera una relación entre la edad, inferida a través del tamaño y/o peso, con las tasas de mercurio de los individuos (Deza 1997). Así, zonas donde predomina la minería que utiliza $\mathrm{Hg}$ como elemento central para la obtención de oro, es muy posible que su fauna acuática esté contaminada y por ende las comunidades que se benefician de ella.

El municipio de Río Quito en el departamento del Chocó, hace varias décadas se ha concentrado como centro de explotación minera en el río Atrato, tanto así que ha desplazado actividades como la pesca y la agricultura. En este contexto y entendiendo la magnitud de la problemática dejada por la minería auroplatinífera; la presente investigación tuvo como finalidad evaluar la presencia de $\mathrm{Hg}$ total en diez especies ícticas utilizadas como autoconsumo por la comunidad.

\section{Metodología}

Área de estudio. La cuenca hidrográfica del río Quito se encuentra localizada en la región suroccidental del departamento del Chocó y tiene una superficie de 166.889,04 hectáreas. De igual forma este accidente orográfico delimitado naturalmente está afectado por los límites políticos de los municipios de Atrato, Istmina, Cértegui, Unión Panamericana, Río Quito y Cantón del San Pablo, 


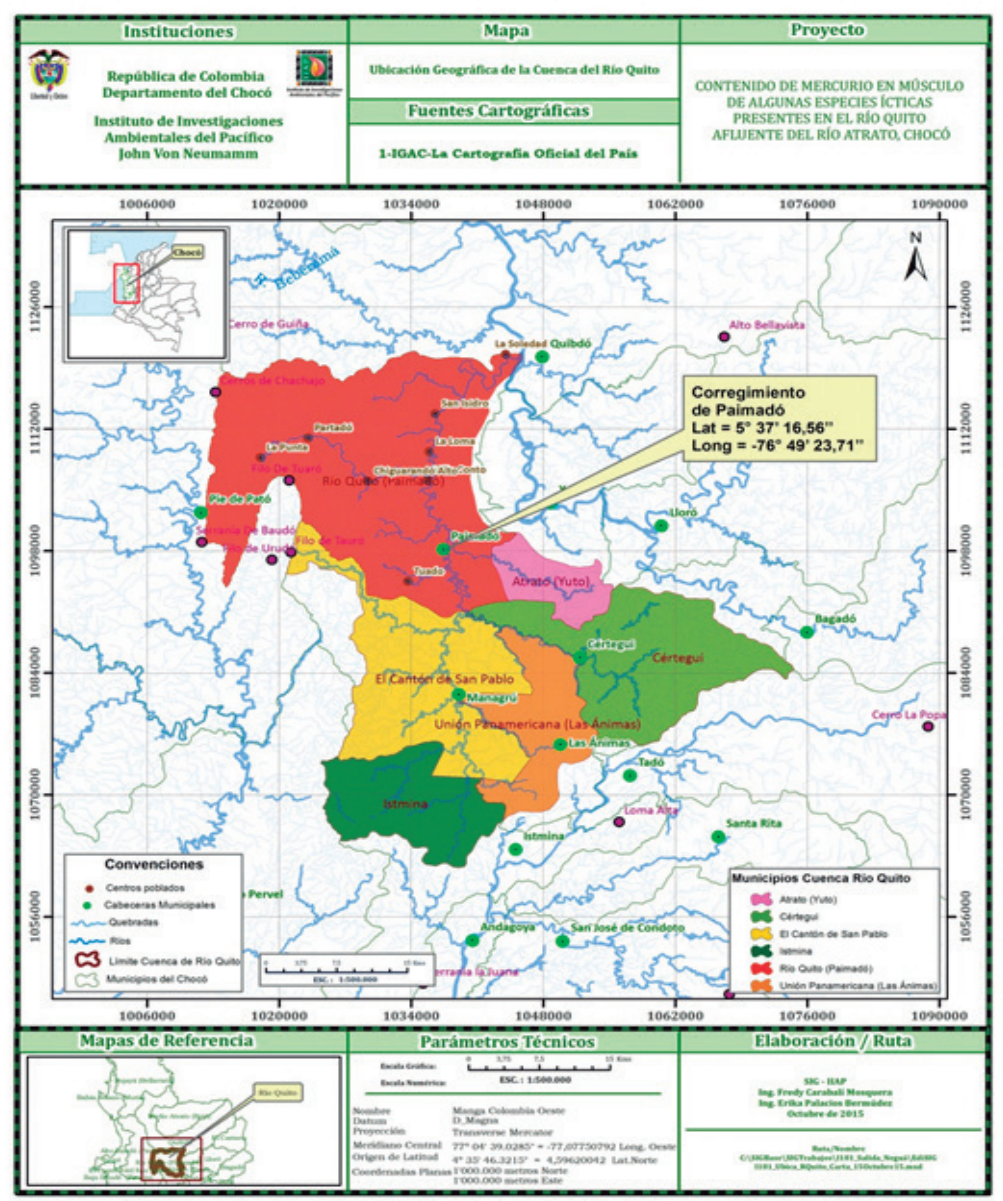

Figura 1. Ubicación y división político-administrativa de la cuenca del río Quito.

la mayor parte del territorio está bajo la jurisdicción del municipio de Río Quito con una superficie de 68.098,56 hectáreas que representa 40,8\% del área total de la cuenca (Tabla 1, Figura 1). Una vez realizado un proceso previo de operación de sistemas de información geográfico (SIG) y basados en la información climática incluida en la Zonificación Ecológica del Pacífico Colombiano y el Mapa de Ecosistemas Continentales Marinos y Costeros de Colombia, se puede observar que en la cuenca del río Quito predominan dos tipos de climas: cálido muy húmedo que representa el $65 \%$ de la superficie de la cuenca y el clima cálido pluvial que representa $34,5 \%$ ambos presentes en todos los municipios que conforman la cuenca.

Esta cuenca tiene 11 tipos de relieves, agrupados a su vez en seis formas de paisajes (cuerpos de agua, lomerío, lomas, montañas, planicies y valles) y geológicamente está compuesta por dos grandes grupos de formaciones geológicas: uno

Tabla 1. Relación superficial en hectáreas de los municipios presentes en la cuenca del río Quito

\begin{tabular}{lrr}
\hline \multicolumn{1}{c}{ Municipio } & Área (Ha) & (\%) \\
\hline Istmina & $16.984,77$ & 10,2 \\
Cértegui & $28.319,51$ & 16,9 \\
El Cantón de San Pablo & $31.436,13$ & 18,8 \\
Río Quito & $68.098,56$ & 40,8 \\
Atrato & $7.320,87$ & 4,4 \\
Unión Panamericana & $14.729,20$ & 8,8 \\
Total & $166.889,04$ & 100,0 \\
\hline
\end{tabular}


es el de las llamas de formaciones integrado por siete formaciones en la cuenca que comprende desde la formación Istmina hasta la formación Quibdó; el otro grupo se conoce como depósitos aluviales integrado por terrazas aluviales, terrazas acuíferas y terrazas consolidadas.

Métodos. Las capturas de los ejemplares se realizaron mediante técnicas artesanales con atarraya y trasmallo. Las muestras fueron depositadas en bolsas plásticas esterilizadas y marcadas con sus respectivos datos (nombre científico y común, lugar de captura y estado en que se encontró el pez) y se situaron en una nevera de fibra con abundante hielo, conservándolas así en una temperatura de $-20^{\circ} \mathrm{C}$ (Escobar-Sánchez 2010). Por último, estas fueron enviadas al laboratorio GAIA de la Universidad de Antioquia, Medellín, donde se determinó los niveles de $\mathrm{Hg}$ presente en el músculo del pez analizado por medio de un proceso de espectrometría de absorción atómica de vapor en frío. La clasificación trófica de las especies se realizó mediante la revisión bibliográfica, siguiendo las descripciones de Maldonado-Ocampo et al. (2012).

\section{Resultados y discusión}

Análisis del contenido de mercurio en peces. Los análisis de mercurio aplicados a 10 especies ícticas usadas como autoconsumo por la comunidad de Río Quito, evidencian que el total de estas muestras contienen mercurio total. Obtuvieron las concentraciones más altas Pseudopimelodus schultzi $(0,76 \mu \mathrm{g} / \mathrm{g})$, Pimelodella chagresii $(0,62$ $\mu \mathrm{g} / \mathrm{g})$, Pimelodus punctatus $(0,45 \mu \mathrm{g} / \mathrm{g})$ mientras Leporinus muyscorum $(0,07 \mu \mathrm{g} / \mathrm{g})$, Astyanax fasciatus $(0,16 \mu \mathrm{g} / \mathrm{g})$, Trachelypterus fisheri $(0,17$ $\mu \mathrm{g} / \mathrm{g})$ y Sturisomathictys tamanac $(0.17 \mu \mathrm{g} / \mathrm{g})$, reportaron los valores más bajos (Tabla 2).

Los datos de Hg-T obtenidos en los bagres (Pseudopimelodus schultzi, Pimelodella chagresii y Pimelodus punctatus), se pueden relacionar con dos grandes comportamientos. La conducta bentónica, la cual le facilita a los bagres la búsqueda de elementos zooplantónicos que se ubican en el fondo acuático (sustrato) y que por su naturaleza ecológica son de los primeros en incorporarse a la cadena. La forma de alimentarse también modela el incremento del mercurio en el músculo de los bagres, pues a pesar de que estas especies poseen una conducta omnívora, es importante resaltar que tienen una fuerte preferencia por los elementos de origen carnívoros. En consecuencia con el párrafo anterior, es importante tener en cuenta que cuando el mercurio entra al medio acuático es depositado en los sedimentos y es convertido principalmente en metilmercurio por acción bacteriana mediante un proceso de biometilación, aumentando de este

Tabla 2. Concentraciones de mercurio en peces analizados

\begin{tabular}{llllc}
\hline Nombre científico & Nombre local & Órgano analizado & Parámetro & Concentración $(\boldsymbol{\mu g} / \mathbf{g})$ \\
\hline Pseudopimelodus schultzi & Bagre sapo & Músculo & $\mathrm{Hg}$ total & 0,76 \\
Pimelodella chagresi & Nicuro & Músculo & $\mathrm{Hg}$ total & 0,62 \\
Pimelodus punctatus & Charre & Músculo & $\mathrm{Hg}$ total & 0,45 \\
Astyanax caucanus & Sardina lunareja & Músculo & $\mathrm{Hg}$ total & 0,38 \\
Rhamdia quelen & Barbudo & Músculo & $\mathrm{Hg}$ total & 0,35 \\
Eigenmannia virescens & Lela & Músculo & $\mathrm{Hg}$ total & 0,21 \\
Sturisomathictys tamanac & Guacuco & Músculo & $\mathrm{Hg}$ total & 0,17 \\
Astyanax fasciatus & Sardina rabicolorada & Músculo & $\mathrm{Hg}$ total & 0,16 \\
Leporinus muyscorum & Denton & Músculo & $\mathrm{Hg}$ total & 0,07 \\
Trachelypterus fisheri & Caga & Músculo & $\mathrm{Hg}$ total & 0,17 \\
\hline
\end{tabular}




\section{Bioetnia Volumen 13, 2016}

modo su biodisponibilidad para la biota acuática (Jewet y Duffy 2007, Hassan et al. 2010). Los organismos que contienen metilmercurio son consumidos por el nivel inmediatamente superior en la cadena alimentaria; también los organismos zooplantónicos son consumido por el siguiente nivel en la cadena trófica; de esta manera, las asociaciones acuáticas consumen una mayor concentración de mercurio en cada uno de los niveles sucesivos de la cadena alimentaria. Por lo tanto, pequeñas concentraciones del mercurio se pueden acumular fácilmente en concentraciones potencialmente nocivas en peces (Hassan et al. 2010), y por supuesto en los humanos por el consumo, sobre todo las comunidades que tienen la fauna íctica como base de su alimentación. Otro carácter que también pueda que influya en alta concentración de mercurio en los bagres, es la fisiomorfológica (piel lisa), lo que estaría facilitando la bioacumulación del metal por exposición directa de su piel con el hábitat (benton-sustrato) contaminado con $\mathrm{Hg}$ - $\mathrm{T}$ donde constantemente se mantienen.

Marrugo et al. (2007), Mancera y Álvarez (2006), Olivero et al. (2009) en sus investigaciones sobre los niveles de biocumulación de $\mathrm{Hg}$ en peces en el canal del Dique, encontraron que las especies ícticas de los eslabones más altos de la cadena trófica (consumidores terciarios) como el moncholo y algunos bagres, presentaron las concentraciones más altas. Sumado a esto Mancera-Rodríguez et al. (2006), encontraron que en fuentes hídricas con influencia directa de desechos mineros (río Ité, Pocuné y El Bagre en Antioquia), las especies de peces con mayores concentraciones fueron las asociadas con el fondo de la columna del agua y las pertenecientes a niveles tróficos superiores, como los bagres, que tienen una marcada tendencia bentónica y hábitos carnívoros. De la misma forma Cala (2001), identificó en el río Magdalena y alto río Meta, que los peces de conducta carnívora presentan los valores más alto de $\mathrm{Hg}$ comparados con otros peces de conducta trófica (iliófagas y herbívoras). Lo anterior muestra que el nivel de acumulación de mercurio en los peces no es igual para todos los ejemplares, porque la concentración de este elemento en los peces depende básicamente de la ubicación del organismo en la cadena trófica, hábito alimenticio, la cercanía que tienen estos con los vertimientos mineros y posiblemente el tiempo de permanencia en el medio acuático (longevidad).

Por otro lado, la sumatoria total de los valores de $\mathrm{Hg}$ obtenidos en los peces analizados, arrojaron un valor de 3,34 $\mu \mathrm{g} / \mathrm{g}$, de estos $41,3 \%$ corresponde a dos especies (bagre sapo y charre), los cuales tienen valores por encima de los rangos $0,2 \mu \mathrm{g} / \mathrm{g}$ y $0,5 \mu \mathrm{g} / \mathrm{g}$ (WHO 2000) permitidos para el consumo humano, tanto para mujeres en estado de gestación, como para menores de edad. Tres especies $(35,3 \%)$ (nicuro, sardina lunareja, barbudo, lela) mostraron concentraciones de $\mathrm{Hg}$ menores a los $0,5 \mu \mathrm{g} / \mathrm{g}$ permitidos para el consumo humano, diferente de las poblaciones vulnerables (menores y mujeres embarazadas). Otro 17\% distribuido en 4 especies (caga, guacuco, sardina rabicolorada, denton) presenta valores de $\mathrm{Hg}$ total por debajo de los estándares $(0,2 \mu \mathrm{g} / \mathrm{g}$ y $0,5 \mu \mathrm{g} / \mathrm{g}$ (WHO 2000) permitidos para el consumo de peces con mercurio; $6 \%$ está en el límite para el consumo de peces con mercurio por parte de menores y mujeres embarazada (Tabla 3) (WHO 2000).

Referente a lo anterior, se puede argumentar que es posible que el consumo constante de las especies ícticas que presentan valores de mercurio total entre $0,35 \mu \mathrm{g} / \mathrm{g}$ y $0,76 \mu \mathrm{g} / \mathrm{g}$ puedan aumentar las posibilidades de contaminación a las personas que utilicen este recurso. Además, si se cuenta con los aportes de Marrugo et al. (2007), quienes manifiestan que el consumo de $0,12 \mathrm{~kg}$ de peces contaminado con $\mathrm{Hg}$, sobre todo carnívoros, incrementa el riesgo de envenenamiento a las poblaciones humanas que los consumen. Se puede sustentar que las especies de bagre (bagre sapo y charre) reportadas con mercurio por encima de lo 
Mercurio en músculo de especies ícticas. YD Murillo Asprilla

Tabla 3. Porcentaje de acumulación mercurial

\begin{tabular}{lccc}
\hline \multicolumn{1}{c}{ Especies } & Concentración $\mathbf{\mu g} / \mathbf{g}$ & Sumatoria de valores de $\mathbf{H g}(\%)$ & $\mathbf{\%} \mathbf{~ d e ~} \mathbf{H g}$ \\
\hline Bagres apo & 0,76 & $1,38(41,3)$ & 20 \\
Nicuro & 0,62 & $1,18(35,3)$ & 30 \\
Charre & 0,45 & & \\
Sardina lunareja & 0,38 & & 10 \\
Barbudo & 0,35 & $0,21(6,3)$ & 40 \\
Lela & 0,21 & $0,57(17,1)$ & \\
Caga & 0,17 & & 100 \\
Guacuco & 0,17 & & \\
Sardina rabicolorada & 0,16 & 3,34 & \\
Denton & 0,07 & & \\
Total & & & \\
\hline
\end{tabular}

estipulado, se podrían considerar un riesgo para el consumo diario de las mismas.

Por su parte, los datos de mercurio encontrados en los peces como el caga, guacuco, sardina rabicolorada y denton, permiten sustentar que es posible que el consumo de estas especies tenga menos posibilidades de contaminar a las comunidades que las utilice (sustento alimenticio). Sin embargo, es importante recordar una vez más, que la ingesta diaria permite acumular concentraciones de este metal en los órganos de quienes los consuman.

\section{Conclusión}

Los resultados expuestos en esta investigación actúan como testigos del nivel de contaminación que en la actualidad presenta la cuenca del río Quito, porque al usar el $\mathrm{Hg}$ para la obtención de oro, se aumenta el riesgo de contaminación de los peces y por ende de las poblaciones humanas que normalmente se alimentan del recurso pesquero. Con el análisis de obtención de Hg se presentó una clara tendencia a la acumulación de este metal en las especies omnívoras con tendencias quizás a la carnivoria, donde Pseudopimelodus schultzi $(0,76 \mu \mathrm{g} / \mathrm{g})$, Pimelodella chagresii $(0,62 \mu \mathrm{g} / \mathrm{g}) \mathrm{y}$ Pimelodus punctatus $(0,45 \mu \mathrm{g} / \mathrm{g})$ obtuvieron las concentraciones más altas de $\mathrm{Hg} ; 41,3 \%$ del Hg obtenido en las muestras está por encima de los rangos permitidos para el consumo humano, tanto para mujeres en estado de gestación como para menores de edad.

\section{Literatura citada}

Barbosa AC, de Souza J, Dórea JG Jardim WF, Fadini PS. 2003. Mercury biomagnification in tropical black water, Rio Negro, Barzil. Arch Environ Contam Toxicol. 45 (2): 235-46. URL disponible en: https://www.ncbi.nlm. nih.gov/pubmed/14565582

Bourgoin LM, Quiroga I. 2002. Total mercury distribution and importance of the biomagnification process in rivers of the Bolivian Amazon. pp. 49-67. In: McClain ME. (ed.) The ecohydrology of south American rivers and wetlands. Venice: UNESCO. URL disponible en: https://books.google.com.co/books?id=Ae1OJ-vyKSkC\&pg=PA49\&lpg=PA49\&dq=Total + mercury + distribution+and+importance + of + the + biomagnification+process + in + rivers + of + the + Bolivian+Amazon\&source $=$ bl\&ots $=$ eHVY03vW2r\&sig $=$ WALG30gW39dABmtiqzcMEb2nGQE\&hl=es\&sa $=X \& v e d=0 a-$ hUKEwiBis7 iPvZAhVEtVMKHfxPDtIQ6AEIMzAB $\# \mathrm{v}=$ onepage $\& \mathrm{q}=$ Tota $1 \% 20$ mercury $\% 20$ distribution $\% 20$ and $\% 20$ importance $\% 20$ of $\% 20$ the $\% 20$ biomagnification $\% 20$ process $\% 20$ in $\% 20$ rivers $\% 20$ of $\% 20$ the $\% 20$ Bolivian $\% 20$ Amazon \&f=false

Cala P. 2001. Occurrence of mercury in some commercial fish species from the Magdalena and Meta rivers in Colombia. DAHLIA. 4: 15-9. 


\section{Bioetnia Volumen 13, 2016}

Da Silva DS, Lucotte M, Roulet M, Poirier H, Mengler D, Oliveira Santos E, et al. 2005. Trophic structure and bioaccumulation of mercury in fish of three natural lakes of the Brazilian Amazon. Water Air Soil Pollut. 165 (1-4): 77-94. URL disponible en: https://link.springer. com/article/10.1007/s11270-005-4811-8

Deza NE. 1996. Mercury accumulation in fish from Madre de Dios, a goldmining area in Amazon Basin, Peru. Thesis for Master of Science. Corvallis: Oregon State University.

Escobar-Sánchez O. 2010. Bioacumulación y biomagnificación de mercurio y selenio en peces pelágicos mayores de la costa occidental de Baja California Sur, México. Tesis doctoral en ciencias marinas. La Paz: Instituto Politécnico Nacional Centro Interdisciplinario de Ciencias Marinas. URL disponible en: http://repositoriodigital. ipn.mx/handle/123456789/16358

Gustin MS, Lindberg SE, Austin K, Coolbaugh M, Vette A, Zhang H. 2000. Assessing the contribution of natural sources to regional atmospheric mercury budgets. Sci Total Environ. 259 (1-3): 61-71. URL disponible en: https://www.sciencedirect.com/science/article/pii/ $\underline{\text { S0048969700005568 }}$

Hassan MAA, Ahmed MK, Akhand AA, Ahsan N, Islam MM. 2010. Toxicological effects and molecular changes due to mercury toxicity in freshwater snakehead (Channa punctatus Bloch, 1973). Int J Environ Res. 4 (1): 91-98.

Herrera C, Millones D. 2011. ¿Cuál es el costo de la contaminación ambiental minera sobre los recursos hídricos en el Perú? Informe final. Consorcio de investigación económica y social. Lima: Departamento de Economía, Pontificia Universidad Católica del Perú.

Hylander LD, Pinto FN, Guimarães JR, Meili M, Oliveira LJ, de Castro e Silva E. 2000. Fish mercury concentration in the Alto Pantanal, Brazil: influence of season and water parameters. Sci Total Environ. 261 (1-3): 9-20. URL disponible en: https://www.ncbi.nlm.nih.gov/ pubmed/11036973

Lechler PJ, Miller JR, Lacerda LD, Vinson D, Bonzongo JC, Lyons WB, et al. 2000. Elevated mercury concentrations in soils, sediments, water, and fish of the Madeira River basin, Brazilian Amazon: a function of natural enrichments? Sci Total Environ. 260 (1-3): 87-96. URL disponible en: https://www.ncbi.nlm.nih. gov/pubmed/11032118

Limbong D, Kumampung J, Rimper J, Arai T, Miyazaki N. 2003. Emissions and environmental implications of mercury from artisanal gold mining in North Sulawesi, Indonesia. Sci Total Environ. 302 (1-3): 227-36. URL disponible en: https://www.ncbi.nlm.nih.gov/ pubmed/12526911

Maldonado-Ocampo JA, Usma JS, Villa-Navarro FA, Ortega-Lara A, Pedreros S, Jimenez L, et al. 2012. Peces dulceacuícolas del Chocó Biogeográfico de Colombia. Bogotá: WWf Colombia, Instituto de Investigaciones de Recursos Biológicos Alexander von Humboltd (IAvH), Universidad del Tolima, Autoridad Nacional de Acuicultura y Pesca (AUNAP), Pontificia Universidad Javeriana; 400 pp. URL disponible en: http://d2ouvy59p0dg6k.cloudfront.net/downloads/ peces choco baja.pdf

Mancera-Rodríguez NJ, Álvarez-Leon R. 2006. Estado del conocimiento de las concentraciones de mercurio y otros metales pesados en peces dulceacuícolas de Colombia. Acta Biol Colomb. 1: 3-23. URL disponible en: https://revistas.unal.edu.co/index.php/actabiol/ article/view $/ 27140$

Marrugo J, Lans E, Benítez L. 2007. Hallazgo de mercurio en peces de la ciénaga de Ayapel, Córdoba, Colombia. Rev MVZ. 12 (1): 878-86. URL disponible en: http:// www.scielo.org.co/pdf/mvz/v12n1/v12n1a03.pdf

Olivero-Verbel J, Caballero-Gallardo K, Torres-Fuentes N. 2009. Assessmen of mercury in muscle of fish from Cartagena Bay, a tropical estuary at the north of Colombia. Int J Environ Health Res. 19: 343-55.

Román-Valencia C. 2004. Datos bioecológicos del peje sapo Pseudopimelodus zungaro (Piscess: Psaudopimelodidea) de los ríos Atrato y Vieja, Colombia. Dahlia 7: 29-31. URL disponible en: http://d2ouvy59p0dg6k. cloudfront.net/downloads/peces_choco_baja.pdf

WHO. 2000. Regional Office for Europe 2000. Chapter 6.9. Mercury. Air Quality Guidelines. 2nd ed. Copenhagem: WHO; 15 pp.

WHO. 2007. Guidance for assessing chemical contaminant data for use in fish advisories. Volume 2. Risk assessment and fish consumption limits. $3^{\text {rd }}$ ed. Washington, DC: WHO; 383 pp. URL disponible en: https://www. epa.gov/sites/production/files/2015-06/documents/ volume2.pdf 